FA, Ebvers GC et al. New diagnostic criteria for multiple sclerosis: guidelines for research protocols. Ann Neurol 1983; 13: 227-31.

Hamilton M. Development of a rating scale for primary depressive illness. Br J Soc Clin Psychol 1967; 6: 27896.

Lewis DA, Smith RE. Steroid-induced psychiatric syndromes. A report of 14 cases and a review of the literature. J Affect Disord 1983; 5: 319-32.

Chrousos GA, Kattah JC, Beck RW, Cleary PA. Side effects of glucocorticoid treatment trial. JAMA 1993; 269: 2110-2.

Falk WE, Mahnke MW, Poskanzer DC. Lithium prophylaxis of corticotropin-induced psychosis. JAMA 1979; 241: 1011-2.

\section{¿Ha aumentado la tasa de varones entre los esquizofrénicos húngaros nacidos después de 1945?}

\section{Z. Rihmer, I. Kecskés}

Departamento de Pacientes Internos y Externos de Psiquiatria $N^{o} X I I I$, Instituto Nacional para Psiquiatría y Neurología, Budapest 27, Pf 1281, Hungría

Estudios recientes han demostrado que la incidencia de la esquizofrenia está disminuyendo, al menos en Escocia, Inglaterra, Gales, Irlanda, Dinamarca y Nueva Zelanda, y esta tendencia ha sido más pronunciada entre las mujeres (Geddes et al, 1993; Waddington y Youseff, 1994), lo que ha resultado en una proporción hombre:mujer más alta en las cohortes más jóvenes de pacientes (Iacono y Beiser, 1992).

Recientemente, hemos investigado también la distribución sexual de los pacientes esquizofrénicos en una muestra de pacientes esquizofrénicos con diagnóstico estricto durante un periodo entre el 1 de enero de 1984 y el 31 de diciembre de 1996. Durante estos 13 años, mientras que nuestro departamento psiquiátrico interno atendía a la misma área de captación (aproximadamente, 90.000 habitantes), y los dos psiquiatras principales del departamento eran los mismos, han cumplido los diagnósticos de esquizofrenia (combinados todos los tipos, pero excluido el trastorno esquizoafectivo) del DSM-III, el DSM-III- R y el DSM-IV 272 pacientes internos (185 mujeres y 87 varones). El mayor número de pacientes feme-
Tabla I. Distribución de nacimientos de 272 pacientes esquizofrénicos según diferentes cohortes de nacimiento

\begin{tabular}{lccc}
\hline Cohorte de nacimiento & Hombres, $\mathrm{n}$ & Mujeres, $\mathrm{n}$ & Total, $\mathrm{n}$ \\
\hline $1976-$ & 1 & 0 & 1 \\
$1971-1975$ & 4 & 3 & 7 \\
$1966-1970$ & 6 & 3 & 9 \\
$1961-1965$ & 7 & 5 & 12 \\
$1956-1960$ & 8 & 11 & 19 \\
$1951-1955$ & 14 & 9 & 23 \\
$1946-1950$ & 9 & 18 & 27 \\
$1941-1945$ & 13 & 21 & 34 \\
$1936-1940$ & 10 & 29 & 39 \\
$1931-1935$ & 9 & 26 & 35 \\
$1926-1930$ & 2 & 19 & 21 \\
$1921-1925$ & 1 & 19 & 20 \\
$1916-1920$ & 1 & 11 & 12 \\
$1911-1915$ & 2 & 6 & 8 \\
$1906-1910$ & 0 & 3 & 3 \\
$1900-1905$ & 0 & 2 & 2 \\
\hline
\end{tabular}

ninos puede ser consecuencia -al menos en parte- del hecho de que nuestro departamento admitió sólo mujeres hasta 1989. Ningún paciente esquizofrénico varón en esta muestra había nacido antes de 1911, y cinco mujeres pacientes nacieron entre 1900 y 1910; sin embargo, después de 1975 había nacido un paciente varón, pero ninguna paciente mujer (tabla I). Tomando en consideración sólo los pacientes nacidos después de 1910 (es decir, excluidos los cinco pacientes mujeres nacidos antes de esa fecha), entre 1946 y 1976 han nacido significativamente más esquizofrénicos varones que mujeres, cuando se compara con el periodo de 1911-1945 (varones: $49 / 87=$ $56 \%$, mujeres: $49 / 180=27 \%, \chi^{2}=21,37, \mathrm{gl}=1$, $P<0,001)$.

La tasa relativamente más alta de mujeres entre los esquizofrénicos mayores puede ser consecuencia del hecho de que la duración media de la vida de los varones en Hungría es 7-8 años más corta. Por otra parte, la tasa relativamente superior de hombres entre los esquizofrénicos más jóvenes puede ser reflejo -al menos en parte- de la edad 4-5 años más baja del comienzo y la primera hospitalización en los varones (Angermeyer y Kühn, 1988). Sin embargo, excluidos los pacientes nacidos después de 1970 (cinco hombres y tres mujeres), la diferencia es significativa (hombres: $44 / 82=54 \%$, mujeres: $46 / 177=26 \%, \chi^{2}=18,92, \mathrm{gl}=1, P<$ 
$0,001)$. La tasa de pacientes mujeres:hombres nacidos entre 1911 y 1930 era 55:6 $(=9,2)$; entre 1931 y 1950 , era $94: 41(=2,3)$, y entre 1951 y 1970 , 28:35 $(=0,8)$.

A pesar del hecho de que nuestra muestra contiene relativamente pocos pacientes esquizofrénicos, pero diagnosticados de manera estricta, estos resultados preliminares apoyan los datos sobre la disminución específica de género en la tasa de esquizofrenia (Geddes et al, 1993; Waddington y Youseff, 1994) también en Hungría, e indican que los varones húngaros jóvenes tienen más posibilidades de desarrollar esquizofrenia que las mujeres.

\section{BIBLIOGRAFÍA}

Angermeyer MC, Kühn L. Gender differences in age at onset of schizophrenia. Eur Archives of Psychiatry and Neurological Sciences 1988; 237: 351-64.

Geddes JR, Black RJ, Whalley LJ, Eagles JM. Persistence of the decline in the diagnosis of schizophrenia among first admissions to Scottish hospitals from 1969 to 1988. Br J Psychiatry 1993; 163: 620-6.

Iacono $\mathrm{GW}$, Beiser $\mathrm{M}$. Are males more likely than females to develop schizophrenia? Am J Psychiatry 1992; 149: 1070-4.

Waddington JL, Youseff HA. Evidence for a gender specific decline in the rate of schizophrenia in rural Ireland over a 50-year period. Br J Psychiatry 1994; 164: 171-6.

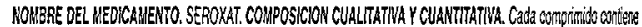

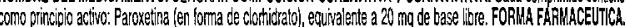

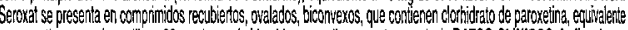

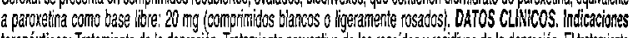

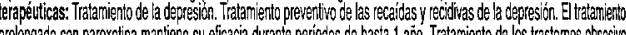
probngaco con paroxetina mantiene su eficacia durante periodes de hasta l año. Tratamiento da los trastonos obsessivo

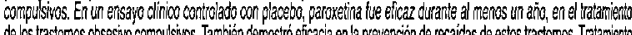

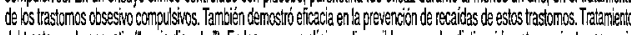

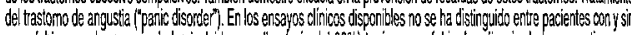

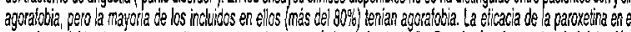

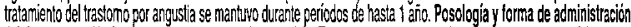

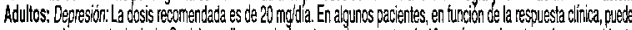

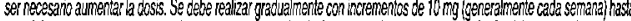

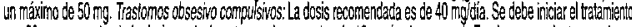

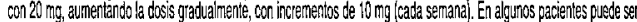
receseno aumentarla drich

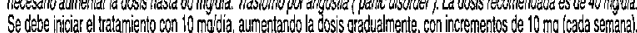

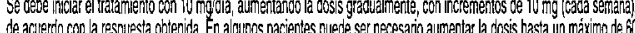

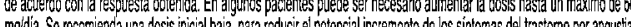

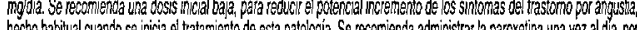

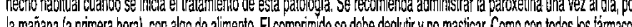

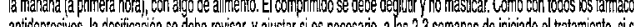

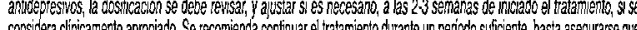

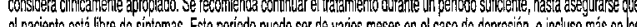

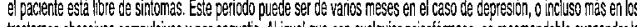

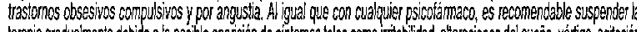

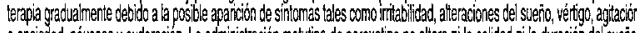

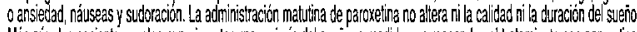
Más aun, los pacientes suelen expenimertar una mejocria del suerio, a mexida que responden al tratamierto con paroxefina

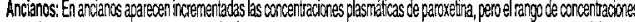

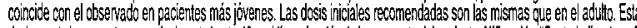

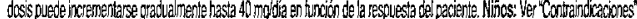

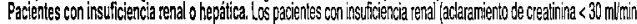

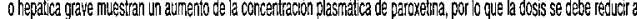
linte infono de mootana a emple

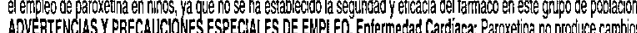
ADVERTENCLAS Y PRECAUCLONES ESPECALLES DE EMPLEO. Entermedad Cardibca: Paroxetina no produce cambios

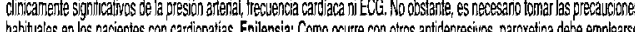

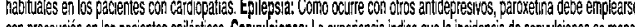

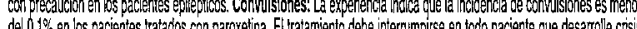

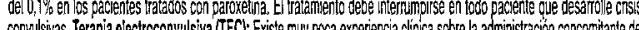

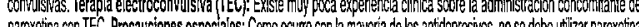

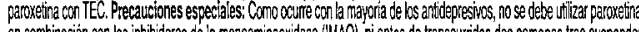

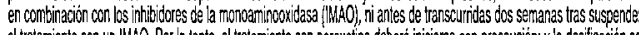

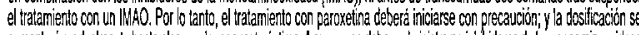

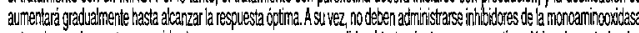
antes de que hayan transcurico dos semanas, una yez suspendido el tratamiento con paroxtetina. Al igual gue tojos los

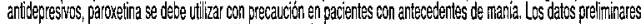

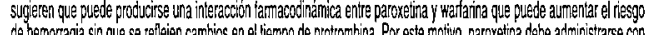
de hemorragia sin que se refleien cantios en el jempo de protorontina. Por este motivo, paroxetna debe adninistrarse con

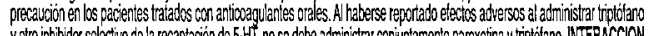

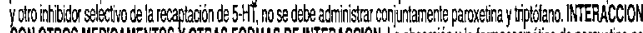

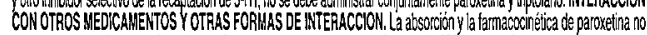

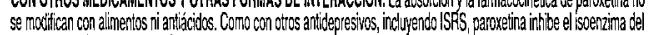
citcromo hepático especiliso $\mathrm{P} 450$ responsable del metabolismo de debrisoquina y espattena. Esto puede conducir a un

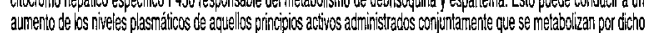

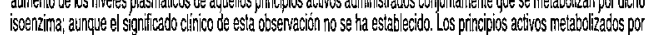

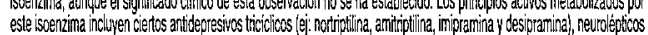

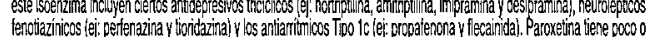

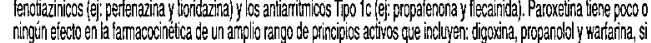

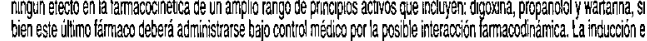

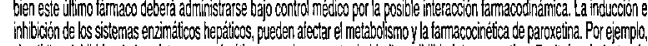

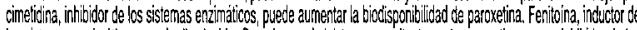

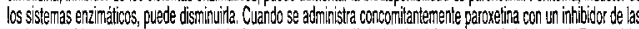

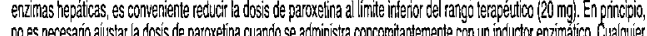

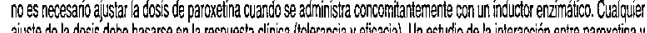

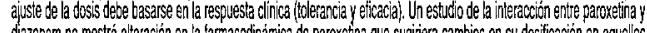

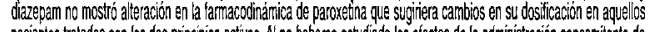

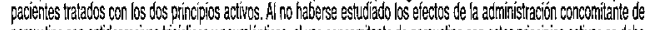

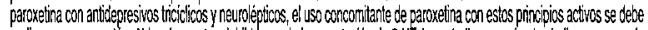

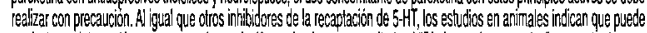

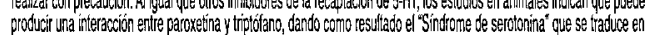

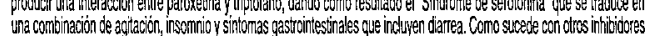

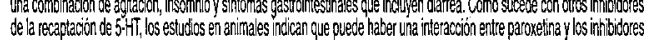

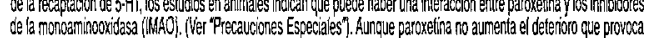

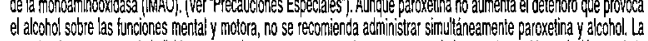

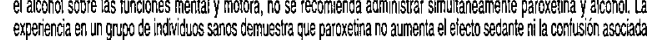

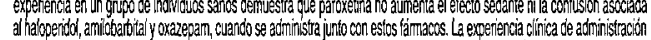

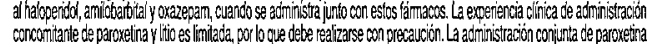

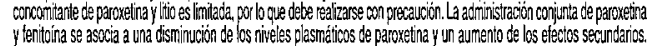

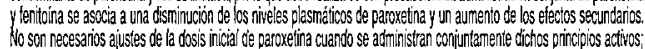

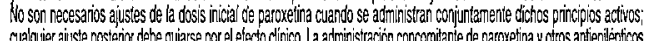

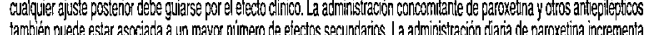

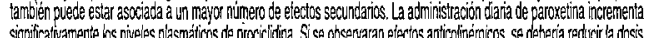

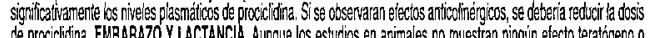
de procidlidina. EMBARAZZO Y LACTANCIA. Aunque los estudios en animales no muestran ningin efecto teratígeno o

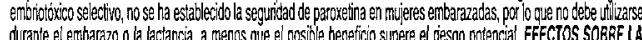

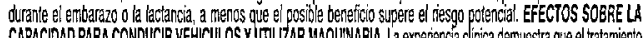

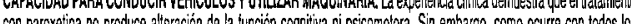

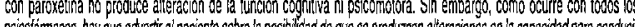

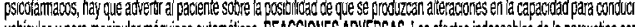

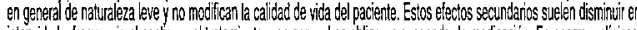

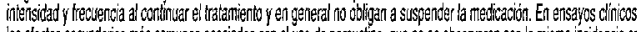

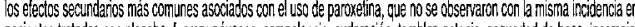

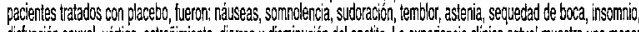

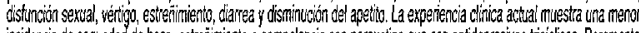
incicencia de secuudad de boca, esterimimiento o somndercia con paroxetina que con antdepresives ticicicos. Raramente

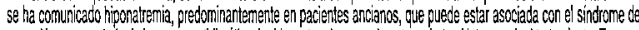

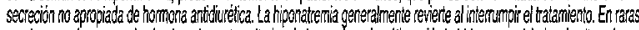
ocasiones se han com unicado elevaciones transionias de los enzimas heoáticos. Ha habido casos aislacos de altelaciones

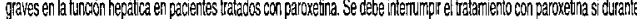

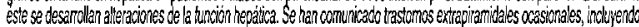
distonia orchacal, en paciertes que a veces padecian de tastonos oculios del moviminento o en aquellos tratados con

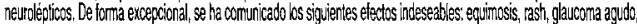
rencon

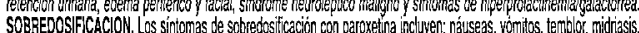

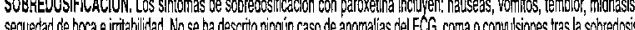

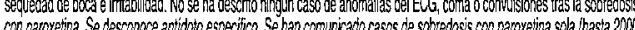

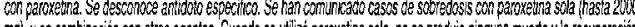

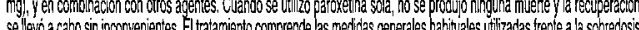

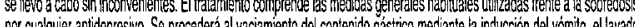

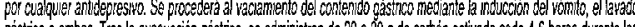

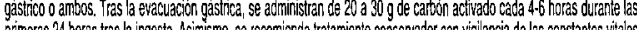

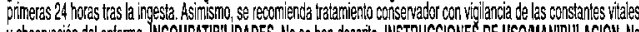

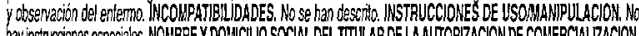

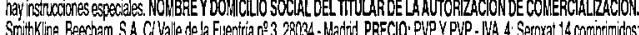

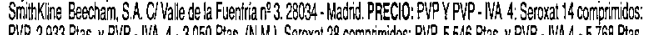

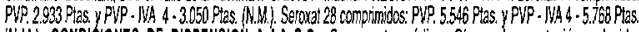

